\title{
Direct detection of the parametrically generated half-harmonic voltage in a Josephson tunnel junction
}

Mygind, Jesper; Pedersen, Niels Falsig; Sørensen, O. H.

Published in:

Applied Physics Letters

Link to article, DOI:

10.1063/1.89061

Publication date:

1976

Document Version

Publisher's PDF, also known as Version of record

Link back to DTU Orbit

Citation (APA):

Mygind, J., Pedersen, N. F., \& Sørensen, O. H. (1976). Direct detection of the parametrically generated halfharmonic voltage in a Josephson tunnel junction. Applied Physics Letters, 29(5), 317-319. https://doi.org/10.1063/1.89061

\section{General rights}

Copyright and moral rights for the publications made accessible in the public portal are retained by the authors and/or other copyright owners and it is a condition of accessing publications that users recognise and abide by the legal requirements associated with these rights.

- Users may download and print one copy of any publication from the public portal for the purpose of private study or research.

- You may not further distribute the material or use it for any profit-making activity or commercial gain

- You may freely distribute the URL identifying the publication in the public portal 


\title{
Direct detection of the parametrically generated half- harmonic voltage in a Josephson tunnel junction
}

\author{
J. Mygind, N. F. Pedersen, and O. H. Soerensen \\ Physics Laboratory I, The Technical University of Denmark, DK-2800 Lyngby, Denmark \\ (Received 4 May 1976)
}

The first direct observation of the parametrically generated half-harmonic voltage in a Josephson tunnel junction is reported. A microwave signal at $f=17.25 \mathrm{GHz}$ is applied to the junction de current biased at zero voltage such that the Josephson plasma resonance $f_{p}=f / 2$. Under these conditions a large-amplitude microwave signal is emitted at $f_{p}$ provided the input power exceeds a threshold value. The results are compared to existing theory.

PACS numbers: 74.35. $+x, 74.50 . \mathrm{Tk}, 74.20 . \mathrm{Gh}, 78.70 . \mathrm{Gq}$

This letter reports the first direct observation of parametric excitation of the plasma oscillation in a Josephson tunnel junction. The junction was dc current biased at zero voltage and irradiated by $f=17.25 \mathrm{GHz}$ microwaves. Large-amplitude oscillations were excited at the Josephson plasma frequency when the dc current was adjusted such that the plasma frequency coincided with half the applied frequency $\left(f_{b}=\frac{1}{2} f\right)$. It was observed that the input power should exceed a certain threshold level in order for this parametric excitation of the plasma oscillation to take place. Theoretically, the phenomenon is well understood ${ }^{1,2}$ and the theoretical results have been confirmed in great detail by experiments performed on an electronic Josephson junction analog. ${ }^{1}$ Also, two recent experiments on real tunnel junctions ${ }^{2,3}$ have indirectly supported the theoretical predictions. The parametrically excited plasma oscillations in Josephson junctions have, however, never before been detected directly. Below, a brief outline of the theory ${ }^{1}$ is followed by presentation of the experimental results, and finally a comparison between the theory and the experiment is made.

The tunnel junction may be represented by a circuit model consisting of the parallel combination of a capacitance $C$, a resistance $R$, and an ideal Josephson element carrying current $I_{0} \sin \phi$. Applying a current bias to this circuit with dc component $I_{\mathrm{dc}}$ and a component at frequency $\omega / 2 \pi$ with amplitude $I_{M}$, the circuit equation becomes

$$
\frac{\hbar C}{2 e}\left(\ddot{\phi}+\frac{1}{R C} \dot{\phi}+\frac{2 e I_{0}}{\hbar C} \sin \phi\right)=I_{\mathrm{dc}}+I_{\mathrm{rt}} \cos \omega t,
$$

where the Josephson voltage-phase relation $V=\hbar \dot{\phi} / 2 e$ has been used. Assuming a solution to $\mathrm{Eq}$. (1) of the form

$$
\phi=\phi_{0}+\phi_{1} \cos (\omega t-\theta)+\delta \phi \exp (-t / R C)
$$

and further assuming that $\delta \phi \ll 1$ and $\phi_{1}^{2} \ll 1$, Eq. (1) leads to

$$
\omega_{p}^{2}\left[1-(1 / 2 Q)^{2}-\phi_{1} \tan \phi_{0} \cos (\omega t-\theta)\right] \delta \phi+\delta \ddot{\phi}=0,
$$

where the plasma frequency $\omega_{p}=\left[\left(2 e I_{0} / \hbar C\right) \cos \phi_{0}\right]^{1 / 2}$, $\phi_{0}=\sin ^{-1}\left(I_{\mathrm{de}} / I_{0}\right)$, and the quality factor $Q=\omega_{\mathrm{p}} R C$ have been introduced. The phase amplitude $\phi_{1}=2 e V_{\mathrm{r}} / \hbar \omega$, where $V_{\mathrm{rg}}$ is junction voltage amplitude at $\omega$, is essentially proportional to $I_{\mathrm{rr}}$. It is known that Eq. (3), which has the form of a Mathieu equation, ${ }^{4}$ for certain values of $\phi_{1}$ becomes unstable in the sense that exponentially increasing solutions exist. It may be shown ${ }^{1}$ that the lowest threshold level occurs around $\omega \simeq 2 \omega_{p}$ and that the solution here will contain a large component at frequency $\omega_{p}=\frac{1}{2} \omega$. For large values of $Q$ and for frequencies in the vicinity of $\omega=2 \omega_{p}$, the threshold curve has the form

$$
\phi_{1} \tan \phi_{0}=2\left\{\left[1-\left(\omega / 2 \omega_{p}\right)^{2}\right]^{2}+\left(1 / Q^{2}\right)\left(\omega / 2 \omega_{p}\right)^{2}\right\}^{1 / 2} .
$$

Finally, it has been observed from analog experiments ${ }^{3}$ that for $\omega \gtrsim 2 \omega_{p}$ the component at $\omega_{p}$ increases smoothly as the threshold is exceeded, whereas for $\omega \leqslant 2 \omega_{p}$ the $\omega_{p}$ component jumps discontinuously to a finite amplitude.

The experiment was performed on a $\mathrm{Nb}-\mathrm{Nb}$ oxideSn junction with dimensions $0.1 \times 0.12 \mathrm{~mm}$ and resistance at $4.2 \mathrm{~K}$ of $\sim 2 \Omega$. This type of junction has been described in detail in Ref. 5 . The junction was mounted directly across the input waveguide of a sensitive $X$ band receiver. The applied radiation was fed to the backside of the junction through a loop-terminated coaxial cable either from a 3-5-GHz transistor oscillator or from a $12-18-\mathrm{GHz}$ sweeper。

The experiment was performed in two steps. First, the plasma resonance was located using the transistor oscillator running at $4.32 \mathrm{GHz}$. With the receiver centered at $8.64 \mathrm{GHz}$ the junction-generated second harmonic voltage was detected. The second harmonic is

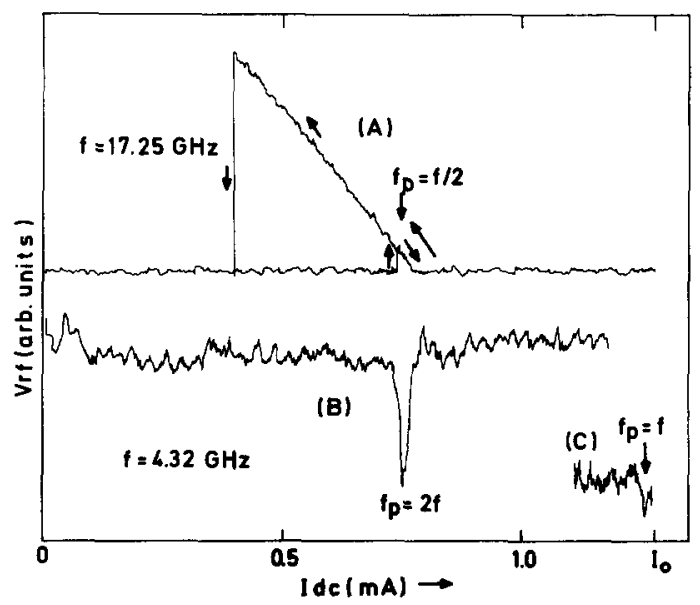

FIG. 1. Detected signal at $8.64 \mathrm{GHz}$ with applied microwaves at (A) $17.25 \mathrm{GHz},(B)$, and (C) $4.32 \mathrm{GHz}$. In (C) the input power level has been reduced $30 \mathrm{~dB}$ relative to (B). $I_{0}=1.275$ $\mathrm{mA}, T=2.72 \mathrm{~K}$. 


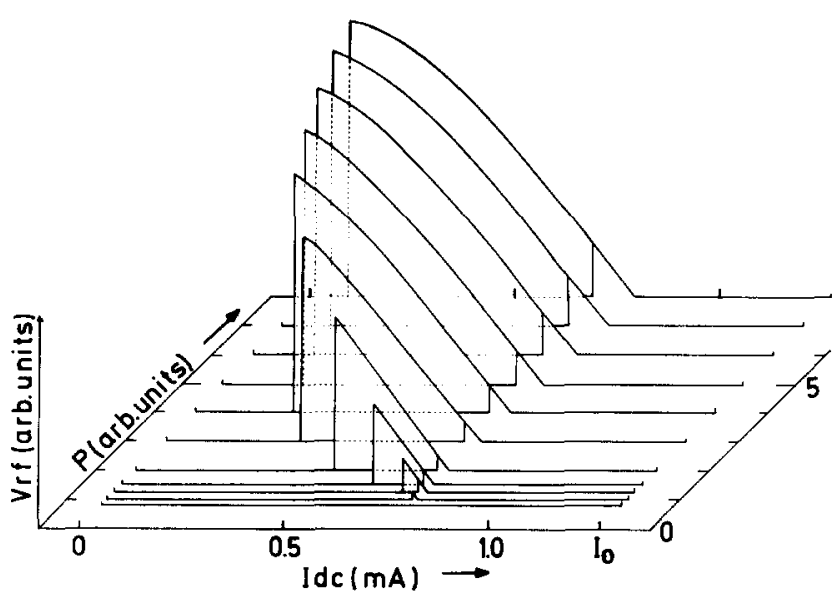

FIG. 2. Detected signal at $8.64 \mathrm{GHz}$ as a function of dc bias current and input power $P$ at $17.25 \mathrm{GHz}$. $T=2.72 \mathrm{~K}$. Reproduced from $X-Y$ recorder traces with noise removed.

known to exhibit two resonances, at $2 \omega=\omega_{p}$ and $\omega=\omega_{p}$, respectively. Both these resonances were observed [Figs. 1(B) and and $1(\mathrm{C})$ ], which allowed us to unambig uously identify $\omega_{p}$. Thus, in trace $B$, the plasma frequency is $8.64 \mathrm{GHz}$ at the bias current $0.755 \mathrm{~mA}$. In this part of the experiment we used a double superheterodyne receiver ${ }^{7}$ which will be described in detail elsewhere. The limiting sensitivity was of order $4 \times 10^{-20} \mathrm{~W}$ in a $1-\mathrm{Hz}$ bandwidth.

In the second part of the experiment a $17.25-\mathrm{GHz}$ signal was applied to the junction and the dc current bias adjusted until the condition $\omega \simeq 2 \omega$, was satisfied. The large magnitude of the parametrically generated subharmonic voltage at $8.64 \mathrm{GHz}$ allowed us to use ordinary video detection.

Typical experimental traces are presented in Fig. $1(\mathrm{~A}), 1(\mathrm{~B})$, and 1(C). Figures $1(\mathrm{~B})$ and 1(C) show the ordinary plasma resonance response as a function of $I_{\mathrm{dc}}$. The peaks correspond to the conditions $2 \omega=\omega_{p}$ and $\omega=\omega_{\text {p }}$ being satisfied.

Figure 1(A) shows a typical parametric half-harmonic response. The detected signal is shown as a function of the dc bias current which has been varied as indicated by the arrows. A characteristic hysteretic behavior is evident. As the bias current is increased (decreasing the plasma frequency) the threshold for parametric excitation is reached, and the junction jumps discontinuously into the excited state at a value of $\omega_{p}>\frac{1}{2} \omega$. A further increase of the current causes the excitation to gradually disappear in a reversible way such that as the current is again decreased $\left(\omega\right.$, now approaches $\frac{1}{2} \omega$ from below) the junction gradually enters the excited state. Finally, when the current is further decreased the excited state persists to much lower values of current (higher $\omega_{p}$ ) than the value where it first appeared, until at some value of current the excitation disappears discontinuously. We have observed this general behavior of the subharmonic voltage also in measurements on an electronic analog.

Figure 2 shows a selection of experimental traces chosen to illustrate the dependence of the parametric subharmonic generation to input power $P$ at $17.25 \mathrm{GHz}$.
As the input power is increased the hysteretic region spans a larger interval of bias current and the maximum detected power increases. The maximum detected power is of order $2 \times 10^{-13} \mathrm{~W}$. An important detail must be pointed out. A finite power exists below which no parametric excitation is observed.

For a fixed input power level the parametric excitation appears at two definite values of bias current depending on whether the current is increasing or decreasing. These current values define two values of $\phi_{0}=\sin ^{-1}\left(I_{\mathrm{dc}} / I_{0}\right)$ and hence the plasma frequencies at the threshold level at that particular input power level.

In order to be able to compare with theory, the quantities $\sqrt{P} \tan \phi_{0} \propto \phi_{1} \tan \phi_{0}$ and $\left(\cos \phi_{0}\right)^{1 / 2} \propto 2 \omega_{p} / \omega$ have been calculated with $\phi_{0}$ determined as described above. Corresponding values have been plotted in Fig. 3. In Fig. 3 are also shown theoretical threshold curves as calculated from Eq. (4). By fitting the theoretical expression to the experimental points the relation between $\sqrt{P}$ and the rf amplitude $\phi_{1}$ as well as the $Q$ of the resonance are determined. In this way we estimate the $Q$ to be between 80 and 100 as illustrated in Fig. 3. This result is consistent with the value deduced from the $2 \omega=\omega_{p}$ resonance peak in Fig. 1(B). The theoretical threshold curves for $Q=50$ and $Q=\infty$ are also shown in Fig. 3. The value of $\phi_{1}$ at the minimum in Fig. 3 is 0.03 , justifying the assumption $\phi_{1}^{2} \ll 1$ of the theory。

Experiment and theory agree closely at low input power levels. At higher input power levels, however, a deviation becomes visible, presumably because Eq. (1) has been linearized in the quantity $\delta \phi$ to produce the Mathieu equation (3). Some deviation at high power levels is then not surprising. The detailed behavior of the subharmonic amplitude cannot be derived in the linear theory discussed here.

We have demonstrated that a tunnel junction, dc current biased at zero voltage, will produce large-amplitude oscillations at the Josephson plasma frequency when irradiated by microwaves at a frequency near twice the plasma frequency, provided that the input level exceeds a certain threshold value. The threshold curve has been determined experimental, and fair

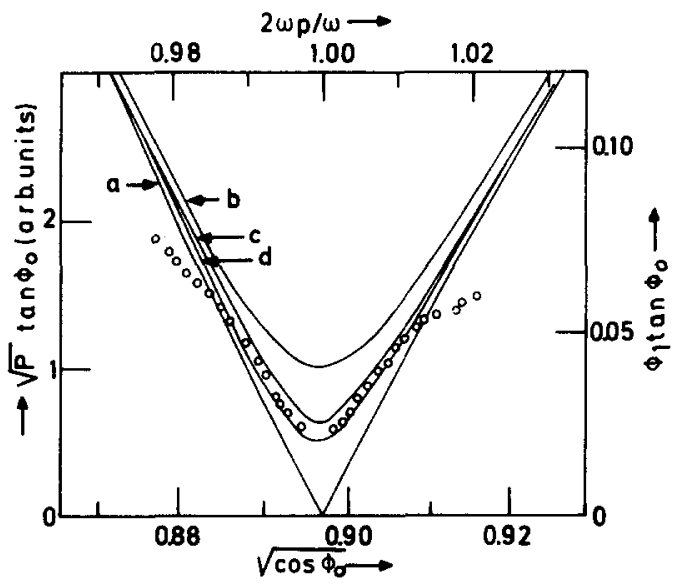

FIG, 3. Experimentally determined threshold values for halfharmonic generation compared to theoretical curves. Curves a-d correspond to $Q=\infty, 50,80$, and 100 , respectively. 
agreement with existing theory has been obtained.

We would like to acknowledge R.D. Parmentier and G. Costabile for supplying the junction used in this experiment, B. Krogh and L. Olsen for technical assistance, and M.R. Samuelsen for stimulating discussions.

${ }^{1}$ N. F. Pedersen, M. R. Samuelsen, and K. Saermark, J. Appl. Phys, 44, 5120 (1973).
${ }^{2}$ A.J. Dahm and D.N. Langenberg, J. Low Temp. Phys. 19, 145 (1975).

${ }^{3}$ C. K. Bak, B. Kofoed, N. F. Pedersen, and K. Saermark, in Proceedings of the Applied Superconductivity Conference, 1974 (Oakbrook, nll., 1975), p. 829.

${ }^{4} \mathrm{M}$. Abramowitz and I. A. Stegun, Handbook of Mathematical Functions (Dover, New York, 1965).

${ }^{5}$ R. D. Parmentier, G. Costabile, P. Rissman, and E. P. Balsamo, J. Low Temp. Phys. 22, 529 (1976).

${ }^{6}$ A.J. Dahm, A. Denenstein, T. F. Finnegan, D. N. Langenberg, and D.J. Scalapino, Phys. Rev. Lett. 20, 859 (1968).

${ }^{7}$ J. Mygind, J. Phys. E 5, 186 (1972).

\title{
Performance of multifilamentary $\mathrm{Nb}_{3} \mathrm{Sn}$ under mechanical load*
}

\author{
D. S. Easton and R. E. Schwall
}

Oak Ridge National Laboratory, Oak Ridge, Tennessee 37830

(Received 10 May 1976)

The critical current of a commercial multifilamentary $\mathrm{Nb}_{3} \mathrm{Sn}$ conductor has been measured under the application of uniaxial tension at $4.2 \mathrm{~K}$ and following bending at room temperature. Significant reductions in $J_{c}$ are observed under uniaxial loading. Results are presented for a monolithic conductor manufactured by the bronze diffusion technique and for cable conductors formed by the tin-dip technique.

PACS numbers: $74.50 . D w, 74.40 . J w, 81.45 .+r, 85.25 .+k$

The construction of large stable superconducting magnets operating at fields of $11 \mathrm{~T}$ or higher presently requires the use of multifilament $(\mathrm{MF}) \mathrm{Nb}_{3} \mathrm{Sn} .{ }^{1}$ Of great concern, however, is the ability of the inherently brittle $\mathrm{Nb}_{3} \mathrm{Sn}$ to operate successfully under the mechanical loads which will be encountered in the construction and operation of large devices. These loads are of two types. The first occurs during winding as the conductor is subjected to twists and bends; the room-temperature bend test simulates this type of stress. ${ }^{2}$ The second type of loading is seen as the device is cooled and energized. The conductor then experiences forces due

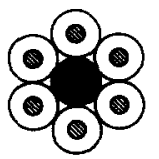

(a)
DIAM $0.60 \mathrm{~mm}$

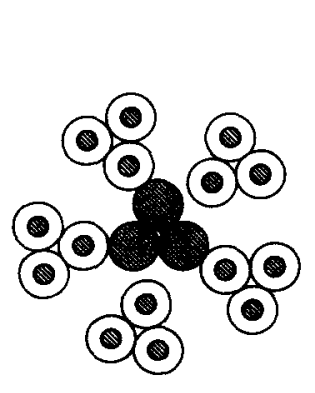

(d)

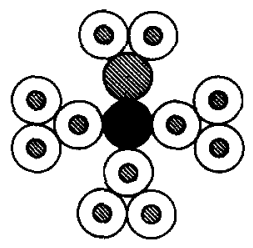

(b)

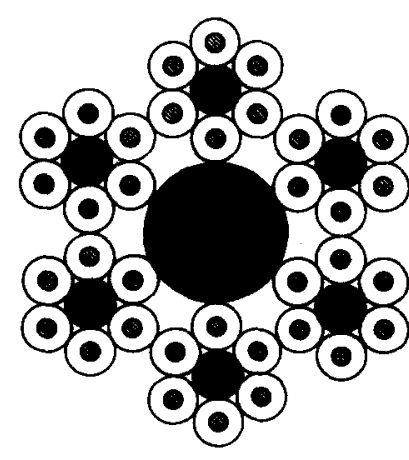

(e)

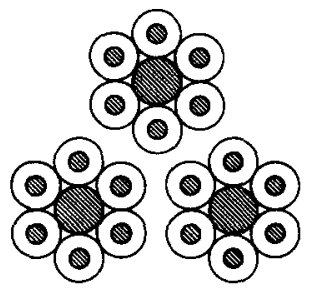

(c)

DIAM $1.14 \mathrm{~mm}$

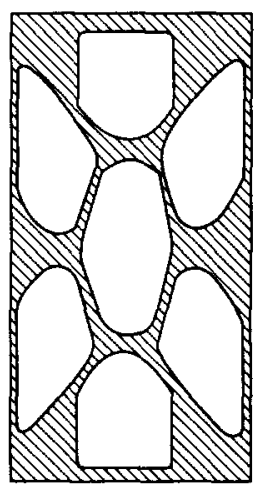

$(f)$
No 3 Sn FILAMENTS IN Cu-Sn MATRIX

COPPER

\section{TUNGSTEN}

OIAM $4.14 \mathrm{~mm}$

DIAM $1.80 \mathrm{~mm}$

$1.0 \times 2.0 \mathrm{~mm}$

FIG. 1. Multifillamentary $\mathrm{Nb}_{3} \mathrm{Sn}$ conductors. 